\title{
STELLAR MASSES AND RADII AS CONSTRAINTS ON STELLAR MODELS
}

\author{
JOHANNES ANDERSEN \\ Copenhagen University Observatory, Brorfeldevej 23, DK-4340 Tølløse, \\ Denmark
}

\begin{abstract}
The current status of empirical data on stellar masses and radii of sufficient accuracy to give constraints on stellar models is reviewed. Results from the best-studied eclipsing binaries can already trace the mainsequence evolution of 1-10 $M_{\odot}$ stars in considerable detail and will be even more useful when supplemented by chemical abundance data.

Taking the deceptively simple question of the observed width of the main sequence as an example, it is shown how careful attention to the details of the data is required to reach robust conclusions about such features of modern stellar evolution models as opacity tables or convective overshooting. Only detailed modelling of specific systems with known masses, radii, and metal abundance constrain the theory strongly enough that a truly critical test is achieved. The same is true when using tidal interactions in binaries (apsidal motion, rotational synchronization, and orbital circularization) as another probe into stellar interiors.
\end{abstract}

\section{INTRODUCTION}

The most basic parameter determining the evolution of star is its mass, followed by its chemical composition. From these initial parameters, stellar evolution theory in principle predicts all observable properties of the star as functions of its age. Observable parameters are, most fundamentally, radius and effective temperature (or luminosity), followed by the photospheric (and chromospheric?) spectrum, rotation, surface inhomogeneities, pulsational frequencies, and perhaps neutrino flux, all subjects thoroughly reviewed at this meeting.

The best test object for stellar models is a star with independently known mass, chemical composition, and age (as input parameters to the model) and also known radius and effective temperature (to be compared with the model). The first and only star for which all these are known is the Sun: It is worth recalling that had our presence on Earth not allowed us to measure the Sun's mass, radius, and luminosity directly and with great accuracy, stellar evolution might never have figured as the subject of a scientific conference. Even today, solar seismo- 
logy and neutrino experiments would be far less informative if the Sun's mass and radius were known to only $\pm 10 \%$ and its metal abundance a mere guess.

For stars other than the Sun, direct determination of mass and radius for comparison with stellar models is only possible in suitable binary systems. However, in order to critically test modern stellar models, the data must above all be accurate: If uncertainties are large enough that all models can be made to fit the data by adjusting one or more free parameters, little new information is gained.

This review will demonstrate that although observable effects of such model features of current interest as opacity tables or convection prescriptions are quite subtle in main-sequence stars, the quality of the modern data, if properly utilized, is quite up to the task of testing these effects. A number of examples how the data can be fully utilized are given. Expressions of sympathy along the way with one or another aspect of particular models should not obscure this basic message.

\section{BRIEF HISTORY AND CURRENT STATUS OF THE DATA}

Comparison of stellar models with masses, radii, and luminosities determined from (eclipsing) binaries is an old subject: Already Schwarzschild (1958) showed a comparison of ZAMS models and B-type main-sequence binaries in the massluminosity diagram. For that time, the agreement (within some two magnitudes) was considered gratifying, but the errors were still large enough that neither composition nor evolutionary effects in the stars were significantly constrained.

Progress in both observations and models allowed Popper et al. (1970) to use the position of well-studied A and F-type binary components in the massradius plane to separate evolved and unevolved stars and use the position of the latter to constrain the metal-abundance parameter $Z$ for the sample. For that value of $Z$, the locus of the unevolved stars in the mass-luminosity diagram constrained the helium abundance, $Y$, to a value close to that currently accepted. Still, apart from any deficiencies of stellar models at that time, uncertain factors remained in the data: No positive evidence existed to show that the stars were in fact on rather than just near the ZAMS; with errors of $5-10 \%$, the mass and radius data did not allow to press the analysis any further. Moreover, it was questionable whether the stars did indeed all have the same metal abundance, and whether the adopted value of $Z$ corresponded to what would be found by direct spectroscopic analysis.

Strömgren (1967) first proposed a detailed simulation of well-observed binaries as the most direct test of the models: For a given (known or assumed) composition, models of the two observed masses are evolved until the observed radii and luminosities of both stars are matched for a common age. As discussed already by Strömgren (1967) and later in more detail by Jørgensen (1978) and Andersen et al. (1991), errors must be no larger that $2 \%$ in mass, $1 \%$ in radius, $2 \%$ in temperature, and $25 \%$ in metal abundance for useful constraints on the models to result. Obtaining data with certifiable errors of this order for the individual stars is far from trivial, however. Only in the most favourable double-lined, eclipsing binaries is this currently possible, and meticulous attention to the choice of system and to the observational material and its analysis is necessary. 
Data for the currently 45 binary systems ( 90 individual stars) for which masses and radii of both components have been determined directly and with errors below $\pm 2 \%$ are listed by Andersen (1991), who also discusses the observational techniques and several applications of the data. We refer to that review and the references given there for further details on the individual binaries and the methods of analysis, and proceed in the following to discuss the relevance of this data set to the subject of this Colloquium.

\section{EVOLUTION WITHIN THE MAIN SEQUENCE}

The degree to which the basic binary parameters, mass and radius, can resolve the fine details of evolution through the main-sequence band is best displayed in the $\log M-\log g$ diagram (Fig. 1). Note that $g$ is here determined directly from $M$ and $R$, independently of uncertainties in parallaxes, photometric calibrations, or spectroscopic line broadening theories; its accuracy is typically an order of magnitude better than possible by such indirect methods. As indicated by the average error bars shown in Fig. 1, evolutionary changes can be resolved to $\sim \pm 5 \%$ of the width of the main-sequence band.

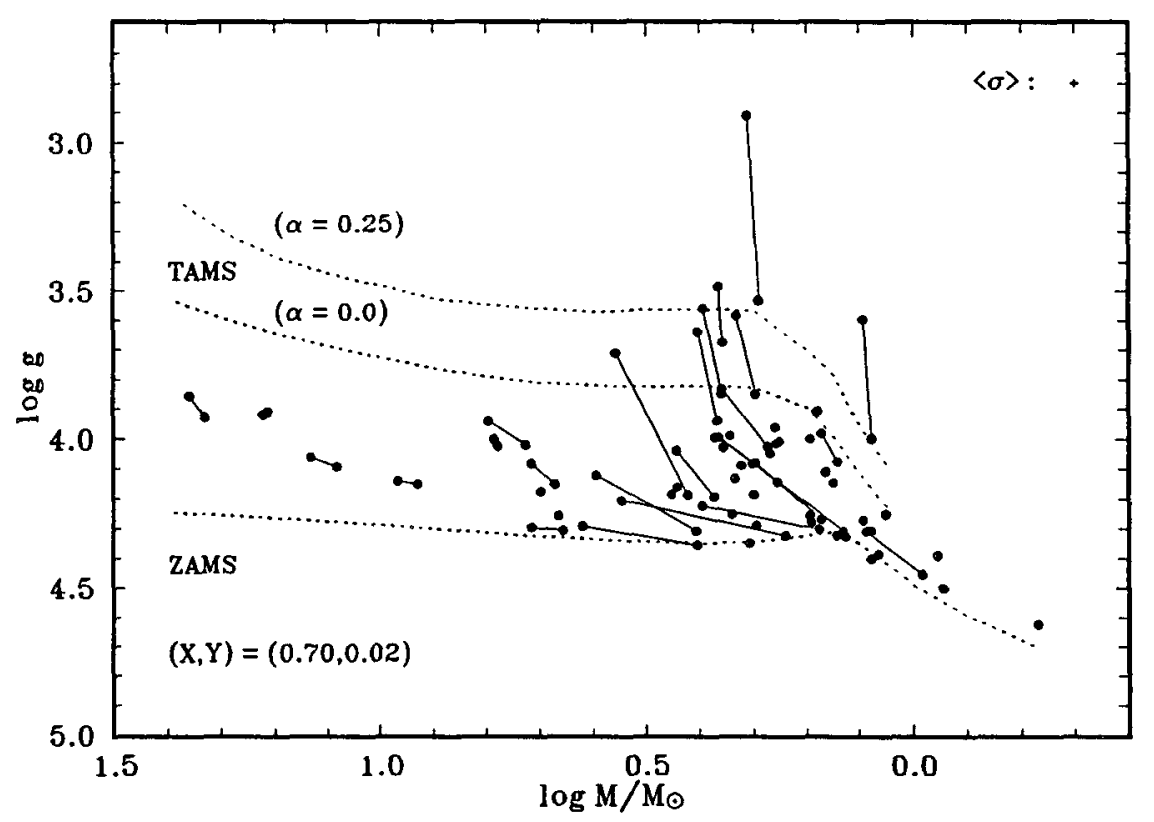

Fig. 1. Mass-gravity diagram for well-determined binary components (Andersen 1991). Lines connect members of the same system, and the average uncertainty of the points is shown. ZAMS and TAMS relations are from Claret \& Gimenez $(1989,1991$ a) 
Assuming no mass loss, evolutionary tracks in the mass-gravity diagram are simple vertical lines along which the stars move (generally upward) with nonuniform speed. Appropriate stellar models must simultaneously predict the correct $\log g$ for both stars in a given system, i.e., isochrones should be parallel to the lines shown in Fig. 1. If the composition of the system is known, this is already a non-trivial test; if $Z$ (and/or $Y$ ) may be freely adjusted, however, a much wider range of models will appear to pass the test. At present, V818 Tau (Popper \& Ulrich 1988) and UX Men (Andersen et al. 1989) are the only bona fide mainsequence systems to which this basic test has been applied; it will be interesting to pursue it further when more metal abundance determinations have been completed (Clausen 1992).

Much of our subsequent discussion is foreshadowed already in Fig. 1, since TAMS lines (the loci for models at the point of central hydrogen exhaustion) are plotted both from models with moderate convective core overshooting $\left(\alpha=d / H_{p}\right.$ $=0.25)$ and from standard models $(\alpha=0.0$; Claret \& Giménez 1989, 1991a). When trying to decide whether a star in this region is still in a slow, central hydrogen-burning phase, we encounter the difficulty that it reverses its direction along the track (the "blue hook") for a brief interval before it proceeds into the Hertzsprung gap on its way to the giant branch. The $\log M-\log g$ diagram (or the equivalent $\log M-\log R$ diagram discussed by Stothers \& Chin 1991) by itself gives no clue to the correct assignment of a given star to any of these overlapping parts of the track. More information - temperatures and/or luminosities - is needed as discussed in the next section.

\section{THE "OBSERVED" WIDTH OF THE MAIN SEOUENCE}

Both major (upwards) opacity revisions in the vicinity of a convective core and the introduction of convective overshooting increase the extent of the cores of stars with masses above $\sim 1.1 M_{\odot}$. The main observable effect for near-mainsequence stars is a widening of the main-sequence band. Much current discussion is centered on this issue, and a notion seems to exist that "the width of the main sequence" is a quantity which is clearly defined and easily determined. The demonstration that this is not so is also a good illustration of some of the subtleties of the use of binary masses and radii, and we devote some attention to the subject here. While the weight of the evidence, in my opinion, at present favours models with mild overshooting over those without, the purpose of the discussion is to point out how the data can be used to discriminate between them. Models change with time; conclusions may change with them.

Open clusters are favourite test objects for stellar models in this mass range (e.g. VandenBerg 1985), and a composite colour-magnitude diagram for many clusters was used by Maeder \& Meynet (1989) to demonstrate the significance of overshooting in these stars and guide the choice of overshooting parameter in their models. The seasoned cluster observer might ask to what extent field stars and binaries could contaminate the colour-magnitude diagrams of open clusters. Similarly, the binary star aficionado used to stars with well-known masses might ask 
whether the real stars do in fact have the same masses as the models used to fit them. Both possibilities are open to observational verification.

Although cluster studies are beyond the scope of this review, we note that our current radial-velocity survey (Nordström \& Andersen 1991) does indeed find some cluster colour-magnitude diagrams to be so affected by binary and field stars as to severely compromise conclusions from fits to the raw diagrams. To provide evidence of a complementary nature (Andersen et al. 1990), we compiled data for well-studied eclipsing binary components in the mass range 1.5-2.5 $M_{\odot}$ (adopting somewhat softer criteria than Andersen 1991). Stars in this range have welldeveloped convective cores, while mass loss on the main sequence remains negligible. Compared with evolutionary tracks for the observed masses in the theoretical HR diagram, far more stars were found in rapid phases of evolution beyond the point of central hydrogen exhaustion (TAMS) than predicted by standard models. In contrast, both acceptable statistics and good fits to individual systems were found with the overshooting models by Maeder \& Meynet (1988).

This interpretation was questioned by Stothers \& Chin (1991) who compared standard models using both the old Cox \& Stewart (1970) and the most recent OPAL opacities (Iglesias \& Rogers 1991, Rogers \& Iglesias 1992) for two values of the metal abundance, the latter models both with and without a small amount of overshooting. Comparing the binary data compiled by Andersen et al. (1990) with the ZAMS and TAMS loci of those models, they concluded that opacity effects alone could, in fact, account for the observed width of the main sequence. Assuming $Z=0.03$, only a small fraction of the stars were found significantly outside the main-sequence band, consistent with the evolutionary lifetimes. They concluded that overshooting is insignificant in these stars.

Apart from the classic problem that "absence of evidence" (here, of overshooting) is not necessarily also "evidence of absence", several questions arise:

First, the metal abundance $Z$ was a free parameter in the fit, but it is unknown whether $Z=0.03$ does, in fact, hold for the real stars. Moreover, when discussing the width of the main-sequence band one must verify whether the ZAMS locus remains consistent with that of observed, unevolved stars of the appropriate metal abundance. The larger radii of metal-rich stars would further raise the ZAMS line in the $\log M-\log g$ diagram (Fig. 1), already uncomfortably close to several of the observed stars. Thus, it is unlikely that $Z=0.03$ is appropriate for the sample as a whole.

In this connection, we note that recent spectroscopic analyses of field B stars (Gies \& Lambert 1992, Kilian 1992) find them to be CNO (and Si) deficient relative to the Sun; see also Nissen's (1992) review in this volume.

Second, the baseline models of Stothers \& Chin (1991), using the outdated Cox-Stewart opacities, are not typical of recent models like those by VandenBerg (1985) or Maeder \& Meynet (1988), which use the more recent Los Alamos Opacity Library (LAOL, Huebner et al. 1977). Fig. 2 compares models for a $2 M_{\odot}$ star using both LAOL and OPAL opacities, each set computed both with and without overshooting, but with otherwise identical input physics. Clearly, in the mass range discussed above, opacity revisions now being seriously considered affect the main-sequence width significantly less than even mild overshooting. 


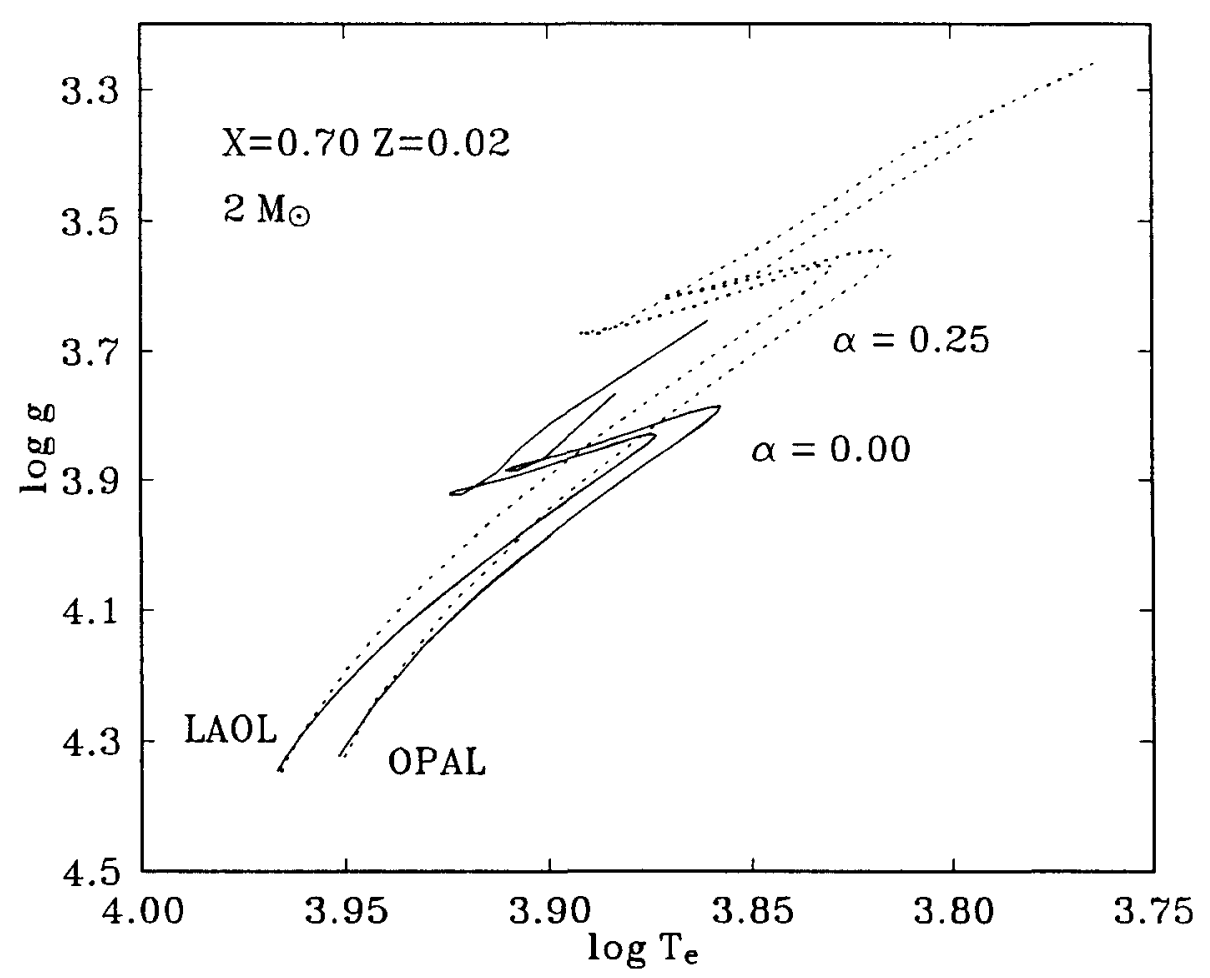

Fig. 2. Evolutionary tracks for a $2 M_{\odot}$ star, with LAOL and OPAL opacities and with and without overshooting (Claret \& Giménez, priv. comm.)

Third, and most important for the present discussion, is the basic ambiguity in assigning stars to slow or rapid evolutionary phases in the TAMS region. It cannot be resolved except by an actual fit of the individual binaries for the observed masses and a consistent age. This is illustrated in Fig. 3, which shows the same VandenBerg models and binary data as discussed by Andersen et al. (1990), but displayed so as to focus attention on the ZAMS and TAMS loci. A casual inspection of the figure might leave the impression that for the high (Hyades) metal abundance of the standard models shown here, only 3-4 stars are significantly outside the main-sequence band. Adding the 44 known binary components located inside this section of the main sequence to the 13 stars shown, this is an entirely plausible fraction.

Respectable agreement between the location of these stars and the tracks for the observed masses (stars and tracks labelled with the same symbols) is also found in most cases. However, essentially all the stars are found to be in the "blue hook" region or in the Hertzsprung gap, i.e. in very rapid phases of evolution. Note that the TAMS may be shifted towards cooler temperatures in the diagram by increasing the metal abundance even further (direction of arrow), but at the same time this makes the fit to the individual tracks even worse. Thus, finding a 


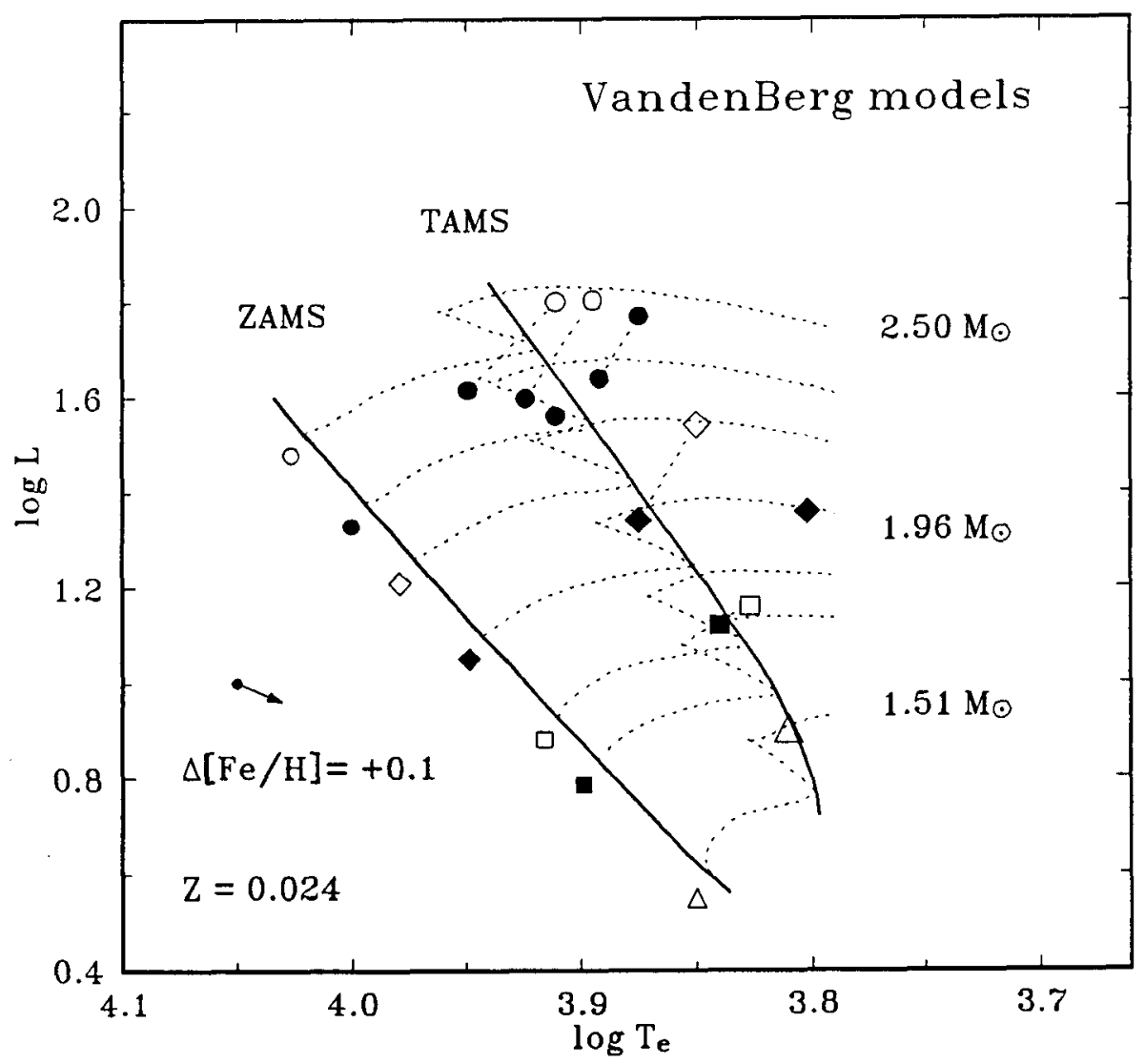

Fig. 3. Evolved binary components $(\log g<3.9)$ of mass $1.5-2.5 M_{\odot}$; from top to bottom: WX Cep, V1031 Ori, SZ Cen, V624 Her A, AI Hya, TZ For B, EI Cep, and RZ Cha AB. Standard evolutionary tracks for the observed masses and a Hyades composition are identified by corresponding symbols. The arrow shows how models shift for the indicated change in $\mathrm{Z}$

star inside "the" main-sequence band in the HR diagram does not prove that it actually is in a slowly-evolving phase unless confirmed by a direct model fit.

In contrast, the solar-metallicity overshooting models shown in Fig. 4 not only place nearly all the stars inside the main-sequence band, but central hydrogen-burning models for the appropriate masses also fit most individual systems within the uncertainties. In this case, assuming a larger metal abundance for these young stars than that of the models (solar) actually improves the fit as shown by the arrow; indeed, the most evolved of these stars, TZ For, has a directly observed $[\mathrm{Fe} / \mathrm{H}]=+0.1$ (Andersen et al. 1991).

We conclude this section by re-emphasizing that its purpose is not to endorse particular models (in fact, ages from Maeder \& Meynet models in this 


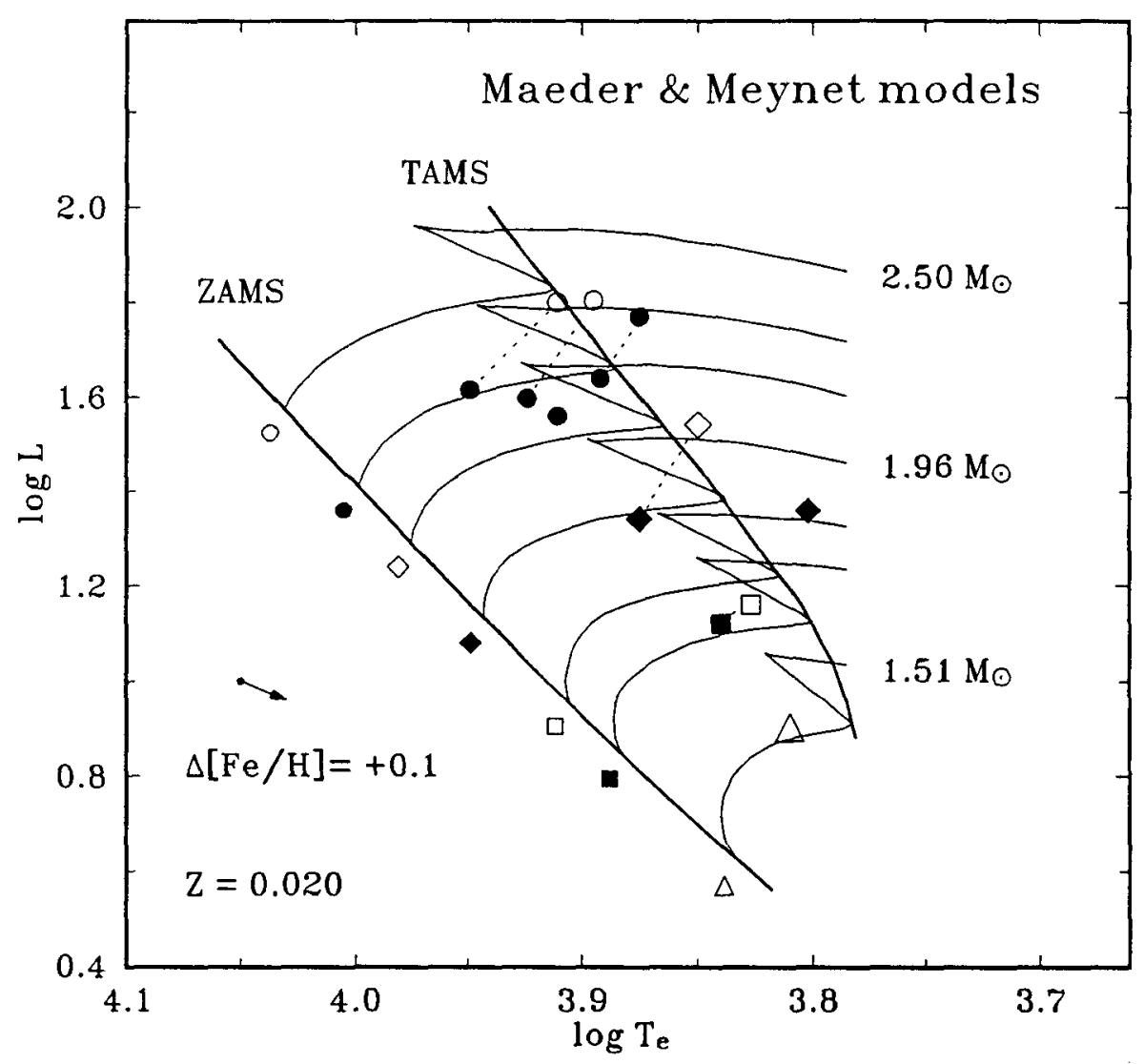

Fig. 4. Same as Fig. 3, but with solar-metallicity overshooting models by Maeder \& Meynet (1988)

mass range require considerable downward revision, as shown by Schaller et al. 1992). The goal has been to show how all available information must be exploited to its full extent for conclusions to be solidly founded.

\section{FITTING INDIVIDUAL SYSTEMS}

The Sun is no doubt the best example of the physical insight which is possible from a refined study of a particularly well-observed star. Models computed for its precisely (and independently) known mass, chemical composition, and age must fit its precise observed radius and effective temperature. Although this is possible by adjusting the assumed helium abundance and mixing-length parameter, reproducing also the observed neutrino flux and oscillation frequency spectrum amounts to a challenge that has not yet been successfully met. 
In stars other than the Sun, Sun-like oscillations have not yet been unambiguously detected (except possibly in $\alpha$ Cen A, Pottasch et al. 1992), and measurable neutrino fluxes are only expected at exceptional occasions (SN 1987A!). In compensation, the requirement that the two stars in a non-interacting binary system be matched by models of the same age and chemical composition provides a significant additional constraint on the models which becomes the most informative if the two stars have appreciably different properties and the observational data are as complete and accurate as modern techniques allow. A few examples are discussed in the following.

Assuming that the metal abundance of a binary system is known together with the component masses, the three additional parameters needed to specify a model for its present state are the mixing-length parameter $\alpha=l / H_{p}$, the helium abundance $Y$, and the age $t$. For the Sun, $t$ is known, and $\alpha$ and $Y$ are adjusted so that the model fits both the radius and the luminosity of the present Sun. For binary components with masses near $1 M_{\odot}$, the solar mixing-length parameter is presumed valid, and $Y$ and $t$ are determined by fitting the radius and luminosity of one of the stars. The test then lies in verifying whether a model with exactly the same parameters matches the radius and luminosity of the other star for its known mass. This is trivially achieved, and no test therefore performed, if the two stars are very similar; the greater the effects of differential evolution, the more significant the test.

As lucidly explained by VandenBerg \& Hrivnak (1985), the 1.2- $M_{\odot}$ binary AI Phe (F7 V + K0 III) is nearly ideal for the purpose, having components of nearly equal mass of which one is still near the main sequence while the other is already on the lower giant branch. In addition to good masses and radii, a photometric metallicity estimate was available, and VandenBerg \& Hrivnak (1985) proceeded to model the system in detail. Inspired by that example, but puzzled by the high helium abundance that was derived, Andersen et al. (1988) provided greatly improved masses $( \pm 0.3 \%)$ and a (spectroscopic) $[\mathrm{Fe} / \mathrm{H}]$ determination for the system. In addition, models for the precise observed masses were specially computed and carefully calibrated, using the VandenBerg (1985) code, including a solar model to determine the mixing-length parameter as outlined above.

The resulting fit is shown in Fig. 5, which merits a few comments: First, the positions of the two observed stars in the diagram are determined directly from the observed temperatures (from multicolour photometry) and radii. For a given temperature, the error in the luminosity is due only to that of the radii, so the error polygons are parallelograms as shown. Second, the range of tracks allowed by the uncertainty in the masses is indicated roughly by the width of the lines; showing only the model for the adopted mass, as is often done, can be deceptive. Third, with models for the observed metal abundance, the helium abundance found by fitting the luminosity of the hotter component is solar: $Y=Y_{\odot}=$ $0.27 \pm 0.01$. Fourth, the age of the hotter component having also been determined in the fit, the models for the subgiant primary were computed up to the same age, i.e. with no free parameters. Age variations of just $\pm 1-2 \%$ would suffice to make this fast-evolving model disagree with the properties of the observed star, but the VandenBerg models pass this most severe test with flying colours. The main 


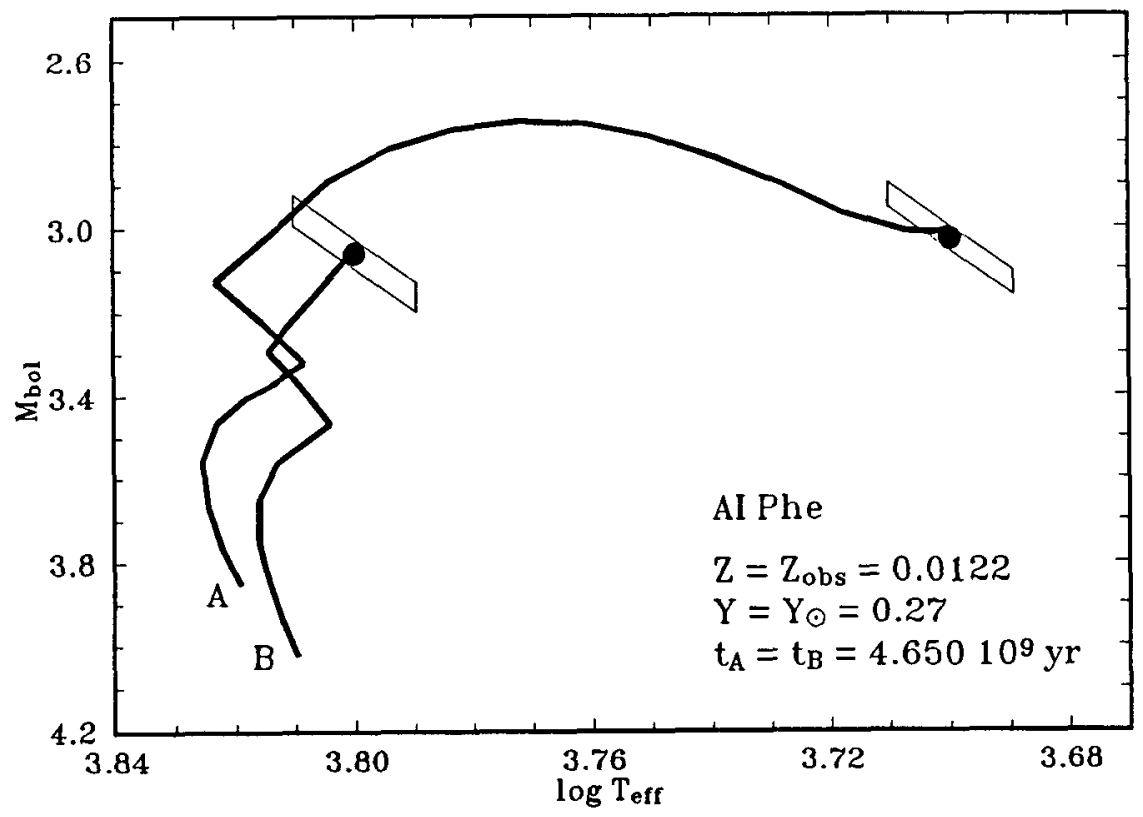

Fig. 5. Evolutionary history of AI Phe (Andersen et al. 1988). Standard VandenBerg (1985) models are shown, for the observed metal abundance, $Y=Y_{\odot}=0.27 \pm 0.01$, and identical ages for both stars. The width of the tracks roughly corresponds to the error in the masses, the width of the error box of star $A$ to an age uncertainty of $\sim \pm 1 \%$

uncertainty in the test is now due to that in the metal abundance (only $\pm 0.1 \mathrm{dex}$ ), not the masses and radii.

By coincidence, the F-type system UX Men was found at the same time to have masses identical to those of AI Phe to within $\pm 0.1 \%(!)$; a metallicity estimate was also close to that for AI Phe. Nevertheless, the stars in UX Men are considerably cooler than the models which fit AI Phe, indicating a higher metal abundance. This was confirmed spectroscopically by Andersen et al. (1989), and VandenBerg (1985) models for the observed $Z$ were again found to be in complete agreement with both observed stars. Including the Hyades binary V818 Hya (Popper \& Ulrich 1988) in the sample shows standard evolution models for near solarmass stars to perform satisfactorily over a range in $Z$ of a factor of two.

A similar study of TZ For, a system very similar to AI Phe but of slightly higher mass $\left(-2 M_{\odot}\right)$ gave a different result (Andersen et al. 1991): Standard models for the observed metal abundance fit the less-evolved F7 IV component well, but match the G8 III giant primary in neither temperature nor age. Moreover, both stars then appear to be in very rapid evolutionary phases, an unlikely if not totally impossible situation. In contrast, models with moderate overshooting (Maeder \& Meynet 1988) are consistent with the position of both stars in the HR 
diagram for the same age. Moreover, the $F$ star is then near the end of its mainsequence evolution and the giant in the core helium-burning ("clump") phase, i.e. both relatively long-lived; clearly a preferable interpretation.

In TZ For, the detailed results permit another test: The mass of the giant component $\left(2.05 M_{\odot}\right)$ is intermediate between the upper mass limits for stars experiencing a helium flash, $2.3 M_{\odot}$ according to standard and $1.85 M_{\odot}$ for overshooting models. The maximum radii of stars undergoing He flash are, however, much larger than in stars above that limit - in TZ For in fact also larger than the Roche lobe. Had TZ For evolved according to standard models, it would have overflowed its Roche lobe in the past and probably suffered extensive mass loss.

A final example of the insight that can be derived from close attention to individual systems in crucial evolutionary phases is SZ Cen (Andersen 1991), the post-TAMS system with equal masses shown by two filled circles in Fig. 3. While increasing the metal abundance beyond the (already high) values of those models may shift the main sequence so as to include at least one of the stars, no increase in $Z$ can get both stars to fit the same track for the observed mass (labelled by the same symbol). The overshooting models (Fig. 4) accomplish this in a natural way.

More detailed comparisons of this type will no doubt provide much useful information on these and other aspects of stellar models. Checking the presumed gradual onset of convective overshooting in (F-type) stars in the mass range 1.1$1.5 M_{\odot}$ should be very informative, as the schematic treatment of overshooting in present models is probably quite unrealistic, especially in small convective cores (Zahn 1991). As discussed above, spectroscopic $[\mathrm{Fe} / \mathrm{H}]$ determinations will be needed to maximize the information that can derived from such comparisons.

\section{EFFECTS OF TIDAL INTERACTION IN BINARY SYSTEMS}

Observable effects of tidal interaction in close binaries include apsidal motion, orbital circularization, and rotational synchronization. They depend very sensitively on the fractional radii of the components. Meaningful comparisons between observed and computed effects require that the radii be determined to $\pm 1 \%$ or so. An extensive review of these effects is outside the scope of this paper (see e.g. Zahn 1992), but a few salient points will be mentioned.

Apsidal motion is one of the classic tests of stellar evolution models, providing a direct measure of the central density concentration in real stars (Schwarzschild 1958). Significant results of the test require well-determined apsidal-motion periods and accurate radii, mass ratios, and rotations to compute the density concentration coefficients $k_{2}$ with sufficient precision. The precise mass and position of the stars within the main-sequence band are needed to provide similarly precise theoretical predictions of $k_{2}$.

Summarizing a persistent effort on this subject, Claret \& Giménez (1991b) concluded that small, but significant discrepancies remained between the computed and observed values of $k_{2}$. In subsequent work, Claret \& Gimenez (1992) find that when convective overshooting, mass loss, and the effects of the known axial rotation of the stars are included in the models, consistency is achieved. Thus, 
improvements in theory and observation are combined in this classic test to yield significant new results on stellar interiors.

For a general review of tidal synchronization and circularization mechanisms, we refer to Zahn (1992). Briefly, their timescales are roughly proportional to $r^{2}$, so rotational synchronization typically occurs 100 times faster than orbital circularization. In stars with radiative envelopes, spin-down of the surface may be decoupled from that of the interior, leading to still shorter synchronization times (Goldreich \& Nicholson 1989). Both timescales depend dramatically on the stellar radii, so binaries with accurately determined properties can be used to test theories of tidal interactions in binaries.

TZ For, discussed above, turns out to be exceptionally informative also in this respect (Andersen et al. 1991): Its period (75 days) is so long that, according to standard tidal theories, several Hubble times would be needed to circularize the orbit. Still, while the orbit is in fact circular, the least-evolved component nevertheless rotates several times faster than the synchronous rate. Attempts to explain TZ For as an exceptional, initially circular case were shown to fail because all known giant binaries in this period range have circular orbits.

The realization that the more massive star in TZ For is in the core heliumburning ("clump") phase provides the key to understanding also its tidal history: At the tip of the giant branch, it was four times larger than now. Since the circularization time varies as $r^{-8}$ it is reduced by a factor $6.510^{4}$, to only a million years. Thus, the brief time spent by the star at the top of the giant branch sufficed for it to circularize the orbit by itself while the companion, still unevolved and with a radiative envelope, continued spinning essentially unaffected. Again, a detailed model fit to the masses and radii of TZ For was the key to understanding the common history of stellar and tidal evolution in this and similar systems.

\section{CONCLUSIONS}

Improvements in the observation and analysis of eclipsing binaries have produced a sample of nearly fifty binaries, listed by Andersen (1991), in which the masses and radii of both components are known with errors of $\pm 2 \%$ or less and effective temperatures are also well-determined. A few of these stars have good spectroscopic metal abundance determinations.

Ways of comparing stellar models with the data can be devised which utilize various subsets of the available parameters mass, radius, temperature/luminosity, and metal abundance. Naturally, the fewer parameters that remain free in the fit, the more informative constraints on the models are obtained. The width of the main-sequence band is discussed as an illustration how detailed model fitting may be needed to get a reliable answer to a simple-minded question. Direct simulation of the evolution of specific systems with complete and accurate data allows a more meaningful test of stellar evolution models than comparison of mean theoretical relations with average properties for less well-observed samples of stars.

The agenda for the immediate future is to provide accurate mass and radius determinations for stars in parts of the main-sequence band that are now poorly 
covered (cf. Andersen et al. 1992), and to complement these with the spectroscopic data that will finally promote the metal-abundance parameter $Z$ from fudge factor to input physics. This effort is well under way (Clausen 1992).

\section{ACKNOWLEDGEMENTS}

It is a pleasure to acknowledge the long and pleasant cooperation with J.V. Clausen, B. Nordström, D.M. Popper, A. Giménez, D. VandenBerg, and A. Claret. Observing time and financial support from ESO, the Danish Natural Science Research Council, The Danish Board for Astronomical Research, the Carlsberg Foundation, and the Smithsonian Institution are gratefully acknowledged.

\section{REFERENCES}

Andersen, J.: 1991, A\&A Rev., 3, 91

Andersen, J., Clausen, J.V., Gustafsson, B., Nordström, B., VandenBerg, D.A.: 1988, $A \& A, 196,128$

Andersen, J., Clausen, J.V., Helt, B.E., Johansen, K.T., Nordström, B., García, J.M., Gimenez, A., Stefanik, R.P., Vaz, L.P.R. 1992, this volume

Andersen, J., Clausen, J.V., Magain, P.: 1989, $A \& A, 211,346$

Andersen, J., Clausen, J.V., Nordström, B., Tomkin, J., Mayor, M.: 1991, $A \& A, 246,99$

Andersen, J., Nordström, B., Clausen, J.V.: 1990, ApJ, 363, L33

Claret, A., Giménez, A.: 1989, A\&AS, 81, 1

Claret, A., Giménez, A.: 1991a, $A \& A S, 87,507$

Claret, A., Giménez, A.: 1991b, A\&A, 244, 319

Claret, A., Giménez, A.: 1992, this volume

Clausen, J.V. 1992, this volume

Cox, A.N., Stewart, J.N.: 1970, ApJS, 19, 243

Gies, D.R., Lambert, D.L. 1992, ApJ, 387, 673

Goldreich, P., Nicholson, P.: 1989, ApJ, 342, 1079 
Huebner, W.F., Merts, A.L., Magee, N.H., Argo, M.F.: 1977, Los Alamos Scientific Laboratory Report, No. LA-6760-M

Iglesias, C.A., Rogers F.J.: 1991, ApJ, 371, 408

Jørgensen, H.E.: 1978, in The HR Diagram (IAU Symp. No. 50), eds. A.G.D. Philip \& D.S. Hayes, Reidel, Dordrecht, p. 433

Kilian, J. 1992, $A \& A$, submitted

Maeder, A., Meynet, G.: 1988, $A \& A S, 76,411$

Maeder, A., Meynet, G.: 1989, $A \& A, 210,155$

Nissen, P.E. 1992, this volume

Nordström, B., Andersen, J.: 1991, ESO Messenger, 63, 34

Popper, D.M., Jørgensen, H.E., Morton, D.C., Leckrone, D.S.: 1970, ApJ, 161, L57

Popper, D.M., Ulrich, R.K.: 1986, ApJ, 307, L61

Pottasch, E.M., Butcher, H.R., Van Hoesel, F.H.J. 1992, this volume

Rogers, F.J., Iglesias, C.A. 1992, ApJS, 79, 507

Schaller, G., Schaerer, D., Meynet, G., Maeder, A. 1992, A\&AS, in press

Schwarzschild, M.: 1958, Structure and Evolution of the Stars, Princeton Univ. Press, Princeton

Stothers, R.B., Chin, C.-w.: 1991, ApJ, 381, L67

Strömgren, B.: 1967, in Modern Astrophysics, ed. M. Hack, Gauthier-Villars, Paris, p. 185

VandenBerg, D.A.: 1985, ApJS, 58, 711

VandenBerg, D.A., Hrivnak, B.J.: 1985, ApJ, 291, 270

Zahn, J.-P. 1991, A\&A, 252, 179

Zahn, J.-P. 1992, in Binaries as Tracers of Stellar Formation, eds. M. Mayor \& A. Duquennoy, Cambridge Univ. Press, in press 